\title{
Modeling the hospital safety partnership preferences of patients and their families: a discrete choice conjoint experiment
}

This article was published in the following Dove Press journal:

Patient Preference and Adherence

26 July 2016

Number of times this article has been viewed

\section{Charles E Cunningham' \\ Tracy Hutchings ${ }^{2}$ \\ Jennifer Henderson ${ }^{2}$ \\ Heather Rimas' \\ Yvonne Chen'}

'Department of Psychiatry and Behavioural Neurosciences, Faculty of Health Sciences, Michael G DeGroote School of Medicine, McMaster University, ${ }^{2}$ Department of Quality and Performance, Hamilton Health Sciences, Hamilton, ON, Canada
Correspondence: Charles E Cunningham Ron Joyce Children's Health Centre, Child and Youth Mental Health Program, 237 Barton Street East, Hamilton, ON L8L 2X2, Canada

Tel +l 9055212100 ext 77307

Fax + I 9055778453

Email cunnic@hhsc.ca
Background: Patients and their families play an important role in efforts to improve health service safety.

Objective: The objective of this study is to understand the safety partnership preferences of patients and their families.

Method: We used a discrete choice conjoint experiment to model the safety partnership preferences of 1,084 patients or those such as parents acting on their behalf. Participants made choices between hypothetical safety partnerships composed by experimentally varying 15 four-level partnership design attributes.

Results: Participants preferred an approach to safety based on partnerships between patients and staff rather than a model delegating responsibility for safety to hospital staff. They valued the opportunity to participate in point of service safety partnerships, such as identity and medication double checks, that might afford an immediate risk reduction. Latent class analysis yielded two segments. Actively engaged participants (73.3\%) comprised outpatients with higher education, who anticipated more benefits to safety partnerships, were more confident in their ability to contribute, and were more intent on participating. They were more likely to prefer a personal engagement strategy, valued scientific evidence, preferred a more active approach to safety education, and advocated disclosure of errors. The passively engaged segment $(26.7 \%)$ anticipated fewer benefits, were less confident in their ability to contribute, and were less intent on participating. They were more likely to prefer an engagement strategy based on signage. They preferred that staff explain why they thought patients should help make care safer and decide whether errors were disclosed. Inpatients, those with immigrant backgrounds, and those with less education were more likely to be in this segment.

Conclusion: Health services need to communicate information regarding risks, ask about partnership preferences, create opportunities respecting individual differences, and ensure a positive response when patients raise safety concerns.

Keywords: hospital safety, patients, partnerships, preferences, discrete choice conjoint experiment

\section{Introduction}

The social and economic costs associated with medical errors are staggering. ${ }^{1}$ Reducing accidents and errors is a major focus of hospital quality programs and an international policy imperative. ${ }^{2}$ Engaging patients is increasingly recognized to be an important component of safety initiatives ${ }^{3-5}$ and a logical extension of efforts to involve patients in the health care process. ${ }^{6}$ Patients are present at the point of care where errors may occur, able to detect medically relevant incidents, ${ }^{7}$ concerned about their safety, ${ }^{8-10}$ and 
motivated to improve the quality of the care they receive. ${ }^{11}$ Patients have been encouraged to reduce hospital-acquired infections by cleaning their hands and reminding service providers to observe hand hygiene protocols. ${ }^{12}$ They have been asked to contribute to the prevention of diagnostic errors, ${ }^{13}$ check medications, ${ }^{14,15}$ join safety committees, ${ }^{6,16}$ voice their safety concerns, ${ }^{17}$ and notify health care workers if errors are detected. ${ }^{18}$

The likelihood of engaging patients in safety partnerships varies as a function of the demographics of both patients and their service providers. Younger patients with higher levels of education, greater health literacy, and more accurate information about potential risks prefer a more active role in the delivery of safe care. ${ }^{8}$ Engagement in safety initiatives also varies with the professional background of the health service providers with whom patients must partner. Patients, for example, are much more likely to direct questions regarding hand hygiene to nursing staff than to physicians. ${ }^{12}$ Davis et $\mathrm{al}^{12}$ reported that, across three studies, $90 \%-100 \%$ of participants questioned nurses about hand hygiene, whereas only $32 \%-40 \%$ asked physicians.

Participation in safety partnerships is also linked to the experiences and attitudes of patients. Although many patients believe that they can contribute to safer hospital care, ${ }^{19}$ their willingness to participate in safety initiatives varies as a function of their risk perceptions, ${ }^{3}$ exposure to medical errors, ${ }^{3}$ and beliefs regarding their role in safety. ${ }^{12,20}$ Cognitive models, such as the Theory of Planned Behavior, predict that the intent to participate would reflect expectations regarding the effectiveness of risk reduction strategies, encouragement by significant individuals, and confidence in one's ability to contribute to prevention. ${ }^{11,12}$

The type of safety partnership patients are asked to participate in exerts a strong influence on their engagement. ${ }^{11,19}$ Discharged inpatients would be more comfortable questioning nurses and doctors about medications than asking whether they had cleaned their hands. ${ }^{6}$ Indeed, while $75.2 \%$ reported that they had inquired about medications, only $4.6 \%$ asked whether staff had cleaned their hands. ${ }^{6}$ Patients are reluctant to make comments that might be perceived to challenge the authority or competence of service providers. ${ }^{8,21}$ They are concerned that health service providers may respond negatively, ${ }^{11}$ patients may be labeled as difficult, ${ }^{3}$ their relationship with service providers may be compromised, and the quality of the care they receive may be affected. ${ }^{17}$ Studies of the attitudes of professionals provide some support for these concerns. ${ }^{22}$

Efforts to engage patients in safety initiatives have included badges encouraging patients to ask whether health care providers cleaned their hands, ${ }^{12}$ posters depicting the correct administration of key health care strategies, ${ }^{23}$ or personal requests from health care professionals. ${ }^{12}$ In one study, patients felt that they would be more than twice as likely to ask whether nurses or physicians had cleaned their hands if health care workers provided an invitation. ${ }^{24}$ Although the best strategy seems to be a personal invitation from health care providers, ${ }^{12}$ relative preference for, and effectiveness of, different engagement strategies is not well understood. This issue is one focus of the current study.

Although the mechanisms via which patients might contribute to a reduction in medical errors seem clear, Berger et al concluded that "while patient engagement in safety is appealing, there is insufficient high quality evidence informing real-world implementation. ${ }^{.25}$ Systematic reviews suggest that there is a need for research regarding the effectiveness of efforts to engage patients, the contribution of patients to a reduction in errors, improvements in health outcomes, or the potential risks of engaging patients in safety partnerships. ${ }^{11,12,16,25}$

Ultimately, an effective engagement strategy is a prerequisite to a successful patient safety partnership. To engage patients, the design of safety partnerships needs to be informed by the preferences of potential participants. ${ }^{11,26}$ Although studies have asked patients for feedback on safety initiatives, few have included patients in the design and development process. ${ }^{12}$ Davis et al ${ }^{12}$ reported that only two of 23 studies included in a systematic review indicated that patients had been engaged in the design process.

\section{The current study}

We extend research in this area by using a discrete choice conjoint experiment (DCE) to engage a large sample of patients, or those acting on their behalf, in the design of an approach to hospital safety partnerships. ${ }^{27}$ Although DCEs have been applied to the design of other risk reduction strategies, ${ }^{28}$ this is, to our knowledge, the first application of these methods to the study of hospital safety partnerships. DCEs make a methodological contribution to safety research by engaging the multistage decision strategies likely to influence the intent to participate in real-world safety initiatives ${ }^{29}$ reducing the influence of social desirability biases, ${ }^{30}$ and improving the estimates of the relative value of the individual components of complex safety initiatives. ${ }^{31}$

We explored four general research questions (RQs) and examined six more specific hypotheses (HYPs).

RQ 1: What features of safety partnerships are most important? We examined the relative importance of 
15 safety partnership attributes. In addition to hand hygiene campaigns that have been the focus of considerable research, ${ }^{12}$ we examined relative preferences for partnerships that might include medication and identity double checks or membership on hospital safety committees. Previous studies suggest that, while patients respond positively to safety partnerships focusing on strategies to ensure the accuracy of medication administration, ${ }^{6}$ they are uncomfortable in addressing staff compliance with hand hygiene protocols. ${ }^{6}$ We predicted, therefore, that:

HYP 1. Patients would show a stronger preference for partnerships involving medication double checks than for those asking staff to clean their hands.

Although patients recognize the value of hand hygiene, ${ }^{24}$ they are hesitant to participate in safety partnerships that involve questioning staff. ${ }^{8,21}$ We predicted that:

HYP 2. Participants would prefer partnerships focusing on hand hygiene for patients rather than an approach encouraging patients to remind staff to clean their hands.

Although previous studies do not provide a basis for specific HYPs, we extend research in this area by exploring a set of partnership features that might influence the decision to participate. These included the way in which risk information is communicated to patients, sources of evidence regarding the benefits of safety partnerships, the process via which patients are engaged in safety partnerships, strategies for providing the training needed to participate, processes for reporting errors, and organizational responses to safety concerns.

RQ 2: Are there segments preferring different safety partnerships? Patients hold different attitudes regarding their role in their health ${ }^{32}$ and safety. ${ }^{6,20,33,34}$ A patient-centered approach to safety partnerships needs to reflect these differences. Using latent class analysis, ${ }^{35}$ we identified segments of participants with different safety partnership preferences and examined factors linked to segment membership.

HYP 3. Given previous studies, ${ }^{32,36}$ we hypothesized a segment of participants preferring a more active approach to safety partnerships and a segment preferring a less active role in safety.

Consistent with the Theory of Planned Behavior, ${ }^{3,11}$ we postulated that:

HYP 4. Those preferring a more active role in safety partnerships would anticipate greater benefit, expect more encouragement to participate, and express more confidence in their ability to contribute.
RQ 3: To what extent do patients prefer a collaborative approach to safety? Although the role of patients in hospital safety has been questioned, ${ }^{37,38}$ patients believe they can contribute to the safety of the care they receive and are willing to consider participating in some safety initiatives. ${ }^{6,11,19,21}$ HYP 5, therefore, postulated that:

HYP 5. Participants would prefer an approach in which patients and staff collaborate to improve safety rather than a model relying solely on staff to ensure safe care.

RQ 4: What type of engagement strategy do patients prefer? RQ 4 addressed a gap in the literature regarding the type of engagement strategies patients prefer. Although signs and posters are an integral component of many hospital safety campaigns, ${ }^{39,40}$ surveys suggest that an invitation from health care workers would be more likely to motivate participation in safety campaigns. ${ }^{9}{ }^{12}$ HYP 6 predicted that:

HYP 6. Patients would prefer that staff, rather than posters or signage, encourage participation in hospital safety partnerships.

\section{Methods}

\section{Participants}

This study was approved by the Hamilton Integrated Research Ethics Board. Data were collected in a regional service, which included a pediatric hospital and five affiliated hospitals serving a population of 2.2 million Canadians. In outpatient waiting areas and inpatient rooms, a hospital staff member asked patients or those acting on their behalf (eg, parents of children, partners, or friends) if they would consider participating. If they agreed, a research team member explained the study, obtained electronic consent, and administered the survey on a laptop computer. Of 1,883 approached, 1,609 (85.4\%) agreed to consider participation. A member of the research team presented the study to 1,567 potential participants (42 of 1,609 were called for an appointment before the study was presented). Although 1,475 agreed to participate, 380 were called for service before completing the survey, eight equipment failures occurred, and nine declined to participate. Overall, 1,084 completed the DCE. Our sample size is consistent with the recommendation of 200 participants per segment. ${ }^{31}$ Demographics are summarized in Table 1.

\section{DCE development}

We developed safety partnership attributes in several steps. ${ }^{41}$ Focus groups or individual interviews were conducted with patients $(n=18)$, family members or support persons $(n=6)$, 
Table I Demographic characteristics of the actively engaged and passively engaged segments

\begin{tabular}{|c|c|c|c|c|c|}
\hline \multirow[t]{2}{*}{ Measure } & \multirow[t]{2}{*}{$\mathbf{N}$} & \multirow[t]{2}{*}{$\%$} & \multicolumn{2}{|c|}{ Latent class segment } & \multirow[t]{2}{*}{$\chi^{2}$} \\
\hline & & & Actively engaged & Passively engaged & \\
\hline Sample size & $\mathrm{I}, 084$ & 100 & 73.3 & 26.7 & \\
\hline Sex & & & & & $20.7 * * *$ \\
\hline Male & 285 & 26.3 & 63.2 & 36.8 & \\
\hline Female & 797 & 73.7 & 77.0 & 23.0 & \\
\hline Age, years & & & & & $19.9 * * *$ \\
\hline$\leq 29$ & 171 & 15.8 & 67.8 & 32.2 & \\
\hline $30-49$ & 653 & 60.5 & 77.6 & 22.4 & \\
\hline $50-69$ & 181 & 16.8 & 69.6 & 30.4 & \\
\hline$\geq 70$ & 75 & 6.9 & 57.3 & 42.7 & \\
\hline Setting & & & & & $35.5 * * *$ \\
\hline Outpatient & 1,000 & 92.6 & 75.6 & 24.4 & \\
\hline Inpatient & 80 & 7.4 & 45.0 & 55.0 & \\
\hline Education & & & & & $38.1 * * *$ \\
\hline High school or less & 303 & 28.1 & 60.1 & 39.9 & \\
\hline Some college or higher & 774 & 71.9 & 78.6 & 21.4 & \\
\hline Participant & & & & & $13.0 * * *$ \\
\hline Patient & 333 & 30.8 & 66.1 & 33.9 & \\
\hline Parent/person on behalf of patients & 747 & 69.2 & 76.6 & 23.4 & \\
\hline Patient health & & & & & 3.6 \\
\hline Routine health visit & 338 & 31.4 & 69.8 & 30.2 & \\
\hline Minor health problem & 298 & 27.6 & 74.8 & 25.2 & \\
\hline Moderately serious health problem & 306 & 28.4 & 75.5 & 24.5 & \\
\hline Serious health problem & 102 & 9.5 & 75.5 & 24.5 & \\
\hline Very serious health problem & 34 & 3.2 & 70.6 & 29.4 & \\
\hline Immigrant status & & & & & $8.0^{* *}$ \\
\hline Born in Canada & 922 & 85.5 & 74.9 & 25.1 & \\
\hline Born in other country & 156 & 14.5 & 64.1 & 35.9 & \\
\hline Language & & & & & 2.8 \\
\hline English & 970 & 90.0 & 74.1 & 25.9 & \\
\hline Not English & 108 & 10.0 & 66.7 & 33.3 & \\
\hline
\end{tabular}

Notes: $* * p<0.01$. $* * * p<0.001$.

a Family Advisory Council ( $\mathrm{n}=6)$, staff $(\mathrm{n}=18)$, and physicians $(\mathrm{n}=1)$. The Family Advisory Council consisted of parents, family members, and community representatives who collaborate with health professionals and leaders to promote family-centered care. Staff participating in focus groups or interviews included registered nurses, physiotherapists, occupational therapists, imaging technologists, clinical managers, and environmental aides. Recordings were transcribed and summarized thematically. Next, we identified widely disseminated safety partnerships and attributes of the implementation process that might influence patient decisions (eg, safety partnership decision making or training). Using a consensual process, we narrowed this information to 15 safety partnership attributes. Attributes ranged from the point of care (eg, medication and identity double checks) to policy and governance (safety committee membership). ${ }^{26}$ Each attribute included four levels ${ }^{42}$ selected to combine logically with the levels of other attributes. ${ }^{31,41}$ We included a level depicting the absence of most attributes. ${ }^{31}$

\section{Experimental design and procedure}

Each participant completed 17 choice sets. Each set presented three safety partnership profiles or options (Figure 1). Participants were instructed that "Below are three ways to make health care safer. Click below the option you would prefer." We used a partial profile design to simplify choices and improve the performance of participants. ${ }^{43,44}$ Each profile included the levels of two attributes. Sawtooth Software's experimental design algorithm created the attribute combinations appearing in each choice set. ${ }^{44}$ Given a main effects design, the attribute levels in each profile did not overlap. The survey defined the term "staff" as doctors, nurses, health care providers, and administrative personnel. The term "patients" was defined as patients or their family members. ${ }^{41}$ Participants completed one warm-up task, 17 choice sets, the Safety Partnership Attitudes Questionnaire described in Figure S1, and demographic questions (a median completion time of 13.4 minutes). 
Below are three ways to make health care safer:

Click below the option you would prefer:

\begin{tabular}{|c|c|c|}
\hline Option 1 & Option 2 & Option 3 \\
\hline $\begin{array}{l}\text { Staff encourage patients to ask } \\
\text { about safety }\end{array}$ & $\begin{array}{l}\text { Volunteers encourage patients } \\
\text { to ask about safety }\end{array}$ & $\begin{array}{l}\text { Signs encourage patients to ask } \\
\text { about safety }\end{array}$ \\
\hline $\begin{array}{l}\text { Patients report safety concerns } \\
\text { to staff }\end{array}$ & $\begin{array}{l}\text { Patients report safety concerns } \\
\text { on an internet form }\end{array}$ & $\begin{array}{l}\text { No formal way of reporting safety } \\
\text { concerns }\end{array}$ \\
\hline 0 & 0 & 0 \\
\hline
\end{tabular}

Figure I Example of the choice sets completed by each participant.

Note: Sawtooth Software's experimental design module composed surveys with different survey attribute combinations for each participant.

\section{Other measures}

To explore attitudes that might influence safety partnership preferences, we developed a Safety Partnership Attitudes Questionnaire reflecting the components of the Theory of Planned Behavior, a model linked to participation in safety initiatives. ${ }^{45}$ We composed 33 Likert-type scale questions (strongly disagree to strongly agree) reflecting the Theory of Planned Behavior's five subscales. Attitudes measured the anticipated benefits of patient safety behaviors. Subjective norms measured contextual and social factors encouraging participation in safety partnerships. Perceived behavioral control: self-efficacy reflected confidence in one's ability to contribute to safety partnerships. Perceived behavioral control: barriers reflected factors that might prevent participation in safety partnerships and intent reflected the stated willingness to participate in different safety partnership activities. Questions from the Safety Partnership Attitudes Questionnaire and internal consistency scores from this study appear in the Supplementary material.

\section{Data analysis}

As described elsewhere, ${ }^{46}$ we used a latent class program (Latent Gold Choice 4.5) to group participants with similar safety partnership preferences into classes. Utility coefficients, reflecting preference for the levels of each safety partnership attribute, were estimated for each class. ${ }^{47}$ Three covariates were included in the latent class model: $:^{35,47}$ intent to participate in safety partnerships (from the Safety Partnership Questionnaire), education (high school or less versus some college or higher), and status as a patient versus parent or person acting on behalf of a patient. A maximum likelihood solution with 1, 2, 3, 4, and 5 classes was estimated. To avoid an unrepresentative model, each solution was computed ten times from different starting points..$^{35,47}$
Using Latent Gold's individual utility coefficients, importance scores were derived by converting the range of the utility coefficients of each attribute to a percentage of the total range across attributes. ${ }^{47}$ Importance scores reflect the relative influence of variation in the levels of each safety partnership attribute on choices.

As described elsewhere, ${ }^{46}$ HYPs 5 and 6 were examined by entering Latent Gold Choice 4.5's individual utility coefficients into Sawtooth Software's Randomized First Choice simulator. ${ }^{48,49}$ Simulations predict each participant's response to hypothetical safety partnerships created by combining the levels of several attributes. Across 200,000 iterations estimating two sources of error, the simulator assumes that participants would choose a safety partnership with a combination of attribute levels yielding the greatest utility. ${ }^{48,50}$

\section{Reliability and validity}

We included identical "hold-out" choice sets at positions 7 and 13 in the 17 set sequences. ${ }^{31,41}$ These choices were not used to compute utility coefficients. ${ }^{31}$ Reliability analysis showed that $96 \%$ of participants made identical choices to the two hold-out sets. Simulations based on the remaining choice data predicted the percentage of participants choosing each option. For both hold-out sets, the difference between predicted and recorded choices was $1.4 \%$. This measure, mean absolute error, shows high predictive validity. ${ }^{31}$

\section{Results}

Based on modeling studies, our goal was to identify a latent class model that minimized Bayesian Information Criterion values and yielded an interpretable solution with administratively manageable sample sizes. ${ }^{51} \mathrm{~A}$ two-class model yielded the lowest Bayesian Information Criterion, the lowest Consistent Akaike Information Criterion, and the highest 
entropy value. A -2 bootstrap log-likelihood (LL) difference test indicated that two classes provided a better fit than a oneclass model, $-2 \mathrm{LL}=818.01, P<0.001$. After considering the safety attitudes and preferences mentioned in the "RQ 2: Are there segments preferring different safety partnerships?" section, we labeled the classes as actively engaged (73.3\%) and passively engaged (26.7\%). We begin by presenting safety partnership attributes on which the two latent classes agreed. We then consider the unique preferences, attitudes, and demographics of the two latent classes. Later, preferences are organized into three consensually derived categories: safety partnership options, communicating risk and safety information to patients, and the safety partnership process.

\section{RQ I: What features of safety partnerships are most important? Safety partnership options}

Importance scores (Table 2) show the relative influence of variations in the levels of each attribute on partnership choices. Patient's identity and medication double checks exerted an especially strong influence on choices. Consistent with HYP 1, the opportunity to participate in medication double checks was more important to participants than hand hygiene campaigns (Table 2). Utility coefficients show the strength of preference for the levels of each attribute (Table 3). Both segments preferred that patients and staff double check patient's identity and medication accuracy. Consistent with HYP 2, an initiative encouraging patients to clean their hands was more important than asking patients to remind staff to clean their hands. Both segments preferred signs (versus staff or volunteers) reminding patients to wash their hands and to ask staff if they had washed their hands (Table 3 ).

\section{Communicating risk and safety information to patients}

Disclosure of risks exerted an important influence on choices (Table 2). Both segments preferred that all patients were informed of risks and that staff encourage patients to ask about safety (Table 3).

\section{Safety partnership process}

The safety reporting process and the hospital's response to safety questions exerted a moderate influence on choices (Table 2). Both segments preferred that patients report safety concerns directly to staff and that those reporting concerns were thanked and informed about the hospital's response (Table 3). Although both segments preferred that patients

Table 2 Relative importance of attributes to the actively engaged and passively engaged segments

\begin{tabular}{|c|c|c|c|c|c|c|c|c|c|}
\hline \multirow[t]{3}{*}{ Attribute } & \multirow{2}{*}{\multicolumn{3}{|c|}{ Total }} & \multicolumn{6}{|c|}{ Latent class segment } \\
\hline & & & & \multicolumn{3}{|c|}{ Actively engaged } & \multicolumn{3}{|c|}{ Passively engaged } \\
\hline & $R$ & $M$ & (SD) & $R$ & $M$ & (SD) & $R$ & $M$ & (SD) \\
\hline \multicolumn{10}{|l|}{ Safety partnership options } \\
\hline Medication double checks & I & 11.7 & $(1.1)$ & I & 12.4 & $(0.1)$ & I & 10.0 & $(0.8)$ \\
\hline Identity double checks & 2 & 11.0 & $(1.5)$ & 2 & 11.9 & $(0.2)$ & 3 & 8.7 & $(1.0)$ \\
\hline Asking safety questions & 5 & 7.0 & $(0.7)$ & 8 & 6.6 & $(0.1)$ & 4 & 8.2 & $(0.4)$ \\
\hline Reporting safety concerns & 8 & 6.6 & $(0.5)$ & 9 & 6.3 & $(0.1)$ & 8 & 7.3 & $(0.3)$ \\
\hline Patient hand cleansing & 10 & 6.2 & $(0.9)$ & II & 5.6 & $(0.1)$ & 6 & 7.6 & $(0.5)$ \\
\hline Safety committee membership & 12 & 5.1 & $(0.7)$ & 12 & 4.6 & $(0.1)$ & 10 & 6.2 & $(0.4)$ \\
\hline Reminding staff to clean their hands & 15 & 3.4 & $(0.3)$ & 15 & 3.3 & $(0.0)$ & 13 & 3.6 & $(0.5)$ \\
\hline \multicolumn{10}{|c|}{ Communicating risk and safety information to patients } \\
\hline Disclosing safety risks & 3 & 8.8 & $(0.1)$ & 3 & 8.7 & $(0.0)$ & 2 & 8.9 & $(0.0)$ \\
\hline Safety partnership training & 7 & 6.8 & $(0.4)$ & 4 & 7.0 & $(0.1)$ & II & 6.1 & $(0.3)$ \\
\hline Evidence supporting partnerships & 6 & 6.8 & $(0.1)$ & 6 & 6.9 & $(0.0)$ & 9 & 6.7 & $(0.0)$ \\
\hline Disclosing mistakes & $\mathrm{II}$ & 6.0 & $(1.1)$ & 7 & 6.6 & $(0.2)$ & 12 & 4.3 & $(0.6)$ \\
\hline \multicolumn{10}{|l|}{ Safety partnership process } \\
\hline Response to safety questions & 4 & 7.1 & $(0.4)$ & 5 & 6.9 & $(0.1)$ & 5 & 7.8 & $(0.2)$ \\
\hline Safety reporting format & 9 & 6.4 & $(0.6)$ & 10 & 6.1 & $(0.1)$ & 7 & 7.5 & $(0.4)$ \\
\hline Safety partnership decision making & 13 & 3.7 & $(0.1)$ & 13 & 3.8 & $(0.0)$ & 15 & 3.5 & $(0.1)$ \\
\hline Anonymity & 14 & 3.4 & $(0.1)$ & 14 & 3.3 & $(0.0)$ & 14 & 3.6 & $(0.0)$ \\
\hline
\end{tabular}

Notes: Important scores are grouped into three consensually derived categories: safety partnership options, communicating risk and safety information to patients, and the safety partnership process. Within these categories, attributes are listed according to their importance to the actively engaged segment. Important scores for each participant were derived by converting the range of each attribute's levels to a percentage of the sum of the utility value ranges of all 15 attributes. Higher importance scores show that variations in the levels of that attribute exerted a greater influence on safety partnership choices. The segments with the highest importance score are bolded. $R$, relative rank of importance score; $M$, mean importance score value.

Abbreviation: SD, standard deviation. 
Table 3 Standardized (zero centered) utility coefficients and Z-values reflecting preferences of the actively engaged and passively engaged segments

\begin{tabular}{|c|c|c|c|c|c|}
\hline \multirow[t]{3}{*}{ Attribute } & \multicolumn{4}{|c|}{ Latent class segments } & \multirow[t]{3}{*}{ Wald } \\
\hline & \multicolumn{2}{|c|}{ Actively engaged } & \multicolumn{2}{|c|}{ Passively engaged } & \\
\hline & $U$ & $\mathbf{Z}$ & $\mathbf{U}$ & $\mathbf{Z}$ & \\
\hline Medication double checks & & & & & $87.87 * * *$ \\
\hline No one double checks that medications are correct & -3.83 & -9.68 & -0.94 & -7.85 & \\
\hline Staff double checks that medications are correct & 1.47 & 10.27 & 0.53 & 6.19 & \\
\hline Patients double check that medications are correct & 0.18 & 1.25 & -0.21 & -2.19 & \\
\hline Staff and patients double check that medications are correct & 2.18 & 14.89 & 0.62 & 7.13 & \\
\hline Identity double checks & & & & & $83.94 * * *$ \\
\hline No one double checks if correct patient & -3.75 & -8.96 & -0.80 & -7.05 & \\
\hline Staff double check if correct patient & 1.57 & 10.36 & 0.45 & 5.10 & \\
\hline Patients double check if correct patient & 0.14 & 0.89 & -0.18 & -1.79 & \\
\hline Staff and patients double check if correct patient & 2.04 & 13.26 & 0.52 & 5.84 & \\
\hline Asking safety questions & & & & & $52.23 * * *$ \\
\hline Patients are not encouraged to ask about safety & -2.06 & -16.62 & -0.81 & -7.17 & \\
\hline Signs encourage patients to ask about safety & 0.60 & 8.89 & 0.41 & 4.63 & \\
\hline Volunteers encourage patients to ask about safety & 0.36 & 5.19 & -0.21 & -2.17 & \\
\hline Staff encourage patients to ask about safety & I.II & 15.17 & 0.62 & 6.90 & \\
\hline Reporting safety concerns & & & & & $58.22 * * *$ \\
\hline Patients are not encouraged to report safety concerns & -2.01 & -16.70 & -0.76 & -6.85 & \\
\hline Signs encourage patients to report safety concerns & 0.64 & 9.59 & 0.52 & 6.15 & \\
\hline Volunteers encourage patients to report safety concerns & 0.36 & 5.19 & -0.22 & -2.19 & \\
\hline Staff encourage patients to report safety concerns & 1.01 & 14.16 & 0.45 & 5.10 & \\
\hline Patient hand cleansing & & & & & $58.15 * * *$ \\
\hline No one reminds patients to clean their hands & -1.91 & -16.60 & -0.72 & -6.75 & \\
\hline Signs remind patients to clean their hands & 0.78 & 11.57 & 0.64 & 7.95 & \\
\hline Volunteers remind patients to clean their hands & 0.38 & 5.72 & -0.19 & -1.99 & \\
\hline Staff remind patients to clean their hands & 0.76 & 11.04 & 0.27 & 3.15 & \\
\hline Safety committee membership & & & & & $27.71 * * *$ \\
\hline $0 \%$ of hospital safety committees include patients & $-1.6 \mid$ & -15.25 & -0.75 & -6.87 & \\
\hline $33 \%$ of hospital safety committees include patients & 0.44 & 6.86 & 0.16 & 1.92 & \\
\hline $67 \%$ of hospital safety committees include patients & 0.60 & 9.26 & 0.23 & 2.70 & \\
\hline $100 \%$ of hospital safety committees include patients & 0.58 & 8.22 & 0.35 & 3.83 & \\
\hline Patients reminding staff to clean their hands & & & & & $56.77^{* * *}$ \\
\hline Patients are not told to remind staff to clean their hands & -1.07 & -12.39 & -0.05 & -0.51 & \\
\hline Signs tell patients to remind staff to clean their hands & 0.53 & 8.99 & 0.36 & 4.41 & \\
\hline Volunteers tell patients to remind staff to clean their hands & 0.14 & 2.21 & -0.33 & -3.49 & \\
\hline Staff tell patients to remind staff to clean their hands & 0.40 & 6.66 & 0.01 & 0.12 & \\
\hline Disclosing safety risks & & & & & $143.49 * * *$ \\
\hline Patients are not told about safety risks & -2.13 & -15.90 & -0.80 & -7.54 & \\
\hline Patients decide if they will be told about safety risks & 0.46 & 6.13 & 0.09 & 1.03 & \\
\hline Staff decide if patients will be told about safety risks & -0.40 & -4.75 & 0.03 & 0.37 & \\
\hline All patients are told about safety risks & 2.08 & 23.08 & 0.68 & 8.30 & \\
\hline Safety partnership training & & & & & $79.31 * * *$ \\
\hline Patients do not learn how to make care safer & -2.52 & -13.97 & -0.60 & -5.84 & \\
\hline Patients read how to make care safer & 0.90 & 11.31 & 0.38 & 4.49 & \\
\hline Patients watch a video and read how to make care safer & 0.75 & 8.99 & 0.07 & 0.76 & \\
\hline Patients watch a video, read, and use a checklist & 0.87 & 10.98 & 0.16 & 1.81 & \\
\hline Evidence supporting partnerships & & & & & $72.74 * * *$ \\
\hline No one explains why patients need to help make care safer & -2.28 & -14.99 & -0.63 & -5.86 & \\
\hline Volunteers explain why patients need to help make care safer & 0.31 & 4.14 & -0.26 & -2.58 & \\
\hline Staff explain why patients need to help make care safer & 0.91 & 12.60 & 0.48 & 5.51 & \\
\hline Staff explain that research shows patients need to help make care safer & 1.05 & 13.76 & 0.41 & 4.70 & \\
\hline
\end{tabular}


Table 3 (Continued)

\begin{tabular}{|c|c|c|c|c|c|}
\hline \multirow[t]{3}{*}{ Attribute } & \multicolumn{4}{|c|}{ Latent class segments } & \multirow[t]{3}{*}{ Wald } \\
\hline & \multicolumn{2}{|c|}{ Actively engaged } & \multicolumn{2}{|c|}{ Passively engaged } & \\
\hline & $\mathbf{U}$ & $\mathbf{Z}$ & $\mathbf{U}$ & $\mathbf{Z}$ & \\
\hline Disclosing mistakes & & & & & $124.03 * * *$ \\
\hline Staff do not tell patients about mistakes & -1.75 & -15.14 & -0.45 & -4.64 & \\
\hline Patients decide if they will be told about mistakes & 0.53 & 7.76 & 0.14 & 1.66 & \\
\hline Staff decide if they will tell patients about mistakes & -0.26 & -3.53 & 0.20 & 2.30 & \\
\hline Staff tell patients about all mistakes & 1.47 & 18.29 & 0.11 & 1.12 & \\
\hline Response to safety questions (patients reporting safety concerns) & & & & & $64.08 * * *$ \\
\hline Are not thanked or told what the hospital will do & -2.06 & -16.94 & -0.76 & -7.01 & \\
\hline Are thanked & 0.37 & 5.46 & 0.05 & 0.56 & \\
\hline Are told what the hospital will do & 0.44 & 6.50 & 0.14 & 1.61 & \\
\hline Are thanked and told what the hospital will do & 1.25 & 17.58 & 0.57 & 6.99 & \\
\hline Safety reporting format & & & & & $71.44 * * *$ \\
\hline No formal way of reporting safety concerns & -2.09 & -14.96 & -0.65 & -6.18 & \\
\hline Patients report safety concerns to staff & 0.82 & 10.98 & 0.65 & 7.67 & \\
\hline Patients report safety concerns on a paper and pencil form & 0.56 & 7.79 & 0.04 & 0.44 & \\
\hline Patients report safety concerns on an internet form & 0.70 & 9.66 & -0.05 & -0.49 & \\
\hline Safety partnership decision making & & & & & $68.79 * * *$ \\
\hline Staff decide if patients will be asked to help make care safer & -1.07 & -13.05 & -0.20 & -2.13 & \\
\hline Patients decide whether to help make care safer & -0.45 & -6.78 & -0.23 & -2.55 & \\
\hline Staff and patients together decide if patients will help make care safer & 0.77 & 12.57 & 0.35 & 4.12 & \\
\hline All patients are asked to help make care safer & 0.75 & 11.76 & 0.08 & 0.90 & \\
\hline Anonymity (patients) & & & & & $25.25 * * *$ \\
\hline Reporting safety concerns do not give their names & 0.15 & 2.56 & 0.15 & 1.70 & \\
\hline Reporting safety concerns give names if they want & 0.77 & 12.47 & 0.34 & 3.92 & \\
\hline Have to give their names after reporting safety concerns & -0.08 & -1.35 & -0.22 & -2.28 & \\
\hline Patients reporting safety concerns must give their names & -0.84 & -11.53 & -0.27 & -2.77 & \\
\hline
\end{tabular}

Notes: $U$, zero-centered utility coefficients. Higher utility coefficients reflect a stronger preference. $Z$, Z-scores. Within segments, the attribute level with the highest utility coefficient and $Z$-values are bolded. $Z$-values of 1.96 differ from zero $(P<0.05)$. $* * * P<0.001$.

decide whether to give their names when reporting safety concerns (Table 3 ), variations in the anonymity afforded patients reporting concerns exerted little influence on choices (Table 2). Although utility values suggest that a collaborative approach to safety partnership decisions was preferred (Table 3), variations in the levels of this attribute exerted a limited influence on choices (Table 2).

\section{RQ 2:Are there segments preferring different safety partnerships?}

Actively engaged and passively engaged segments of the two-segment latent class solution were consistent with HYP 3's predictions. We consider differences in these segments subsequently.

\section{Actively engaged}

Covariate analysis suggests that membership in the actively engaged segment was linked to higher education, $P<0.001$, and a greater intent to participate in safety partnerships, $P<0.01$. The status of informants as patients versus those acting on behalf of patients was not associated with segment membership, $P=0.09$. The actively engaged segment was likely to be younger, outpatients, born in Canada (Table 1). Scores from the Safety Partnership Attitudes scale (Table 4) address HYP 4. As predicted, actively engaged participants anticipated greater benefits to partnerships, reported more confidence in their ability to contribute, and expressed a stronger intent to participate. This segment preferred that staff present the research supporting safety partnerships (Table 3). Although they preferred simply reading how to make care safer, they also responded positively to a multicomponent approach, including readings, videos, and checklists. The actively engaged segment preferred that staff, rather than signs, encourage patients to report safety concerns and that staff disclose all mistakes.

\section{Passively engaged}

Membership in the passively engaged segment was associated with lower education and a lower intent to participate in safety partnerships. Participants who were immigrants were more likely to reside in this segment (Table 1). This segment anticipated fewer safety partnership benefits and 
Table 4 Safety partnership attitudes scale scores for the actively engaged and passively engaged segments

\begin{tabular}{|c|c|c|c|c|c|c|}
\hline \multirow[t]{3}{*}{ Content of question } & \multicolumn{4}{|c|}{ Latent class segment } & \multirow[t]{3}{*}{$F$} & \multirow[t]{3}{*}{$\eta^{2}$} \\
\hline & \multicolumn{2}{|c|}{ Actively engaged } & \multicolumn{2}{|c|}{ Passively engaged } & & \\
\hline & M & (SD) & $M$ & (SD) & & \\
\hline Attitudes (benefits) & 29.4 & $(4.8)$ & 28.0 & $(4.9)$ & $16.5 * * *$ & 0.015 \\
\hline Subjective norms & 28.3 & $(4.2)$ & 28.9 & $(4.8)$ & $4.8^{*}$ & 0.004 \\
\hline Perceived behavioral control: self-efficacy & 23.2 & $(3.6)$ & 22.3 & $(4.3)$ & $12.0 * *$ & 0.011 \\
\hline Perceived behavioral control: barriers & 15.5 & $(4.4)$ & 15.4 & $(4.4)$ & 0.3 & 0.000 \\
\hline Intent & 26.9 & $(3.5)$ & 26.0 & $(4.1)$ & $13.8 * * *$ & 0.013 \\
\hline
\end{tabular}

Notes: $\eta^{2}=$ partial eta squared. $* P<0.05$. $* * P<0.01$. $* * * P<0.001$.

Abbreviations: $M$, mean; $S D$, standard deviation.

was less confident in their ability to contribute (Table 4). The utility values show that they preferred staff to decide whether patients were informed of mistakes (Table 3). Importance scores show that the safety partnership decision-making process was among the attributes exerting the least influence on choices. In contrast to the evidence-informed approach preferred by the actively engaged segment, passively engaged participants preferred that staff explain why they (rather than research) felt patients should participate in safety partnerships. Patient representation on hospital safety committees was more important to the passively engaged segment than to the actively engaged segment; they preferred that patients were represented on all safety committees.

\section{RQ 3:To what extent do patients prefer a collaborative approach to safety?}

To explore this question, we simulated each participant's response to three approaches to safety. While utility coefficients examine relative preference for the individual levels of each attribute, simulations capture the complexity of multicomponent, real-world safety initiatives. ${ }^{12} \mathrm{We}$ manipulated the levels of three attributes while holding 12 constant. According to the safety partnership model, patients received safety training that 1) included readings, videos, and checklists. Both patients and staff double checked that 2) staff helped the right patient and 3) medications were correct. According to the staff safety model: 1) patients did not learn to make care safer. Staff double checked that 2) they were helping the right patient and 3) medications were correct. In the control condition, 1) patients did not learn to make care safer, and no one double checked that 2) staff were helping the right patient, and 3) medications were correct. Consistent with HYP 5 simulations predicted that both actively (100\%) and passively engaged (91\%) participants would prefer partnerships with staff rather than a model delegating safety to staff alone (Table 5).

\section{RQ 4: What type of engagement strategy do patients prefer?}

We used Randomized First Choice simulations to address this question and to examine HYP 6's prediction that patients would prefer that staff personally engage them in safety partnerships. We manipulated the levels of four attributes while holding eleven constant. According to the personal engagement model, staff encouraged patients to 1) ask about safety, 2) report concerns, 3) clean their hands, and 4) remind staff to wash their hands. According to the visual engagement model, signs encouraged patients to 1) ask about safety, 2) report concerns, 3) clean their hands, and 4) remind staff to clean their hands. In the control condition, patients were not

Table 5 Randomized first choice simulations: percentage of participants in each segment predicted to prefer different approaches to the design of safety partnerships

\begin{tabular}{|c|c|c|c|c|c|c|c|}
\hline \multirow[t]{3}{*}{ Simulation } & \multirow[t]{3}{*}{ Safety option } & \multirow{2}{*}{\multicolumn{2}{|c|}{ Total sample }} & \multicolumn{4}{|c|}{ Latent class segment } \\
\hline & & & & \multicolumn{2}{|c|}{ Actively engaged } & \multicolumn{2}{|c|}{ Passively engaged } \\
\hline & & $\%$ & SE & $\%$ & SE & $\%$ & $\mathrm{SE}$ \\
\hline \multirow[t]{3}{*}{ Simulation I } & Control & 0.0 & 0.0 & 0.0 & 0.0 & 0.1 & 0.0 \\
\hline & Staff safety model & 2.4 & 0.1 & 0.0 & 0.0 & 8.9 & 0.3 \\
\hline & Safety partnership model & 97.6 & 0.1 & 100.0 & 0.0 & 91.0 & 0.3 \\
\hline \multirow[t]{3}{*}{ Simulation 2} & Control & 0.1 & 0.0 & 0.0 & 0.0 & 0.3 & 0.0 \\
\hline & Visual engagement model & 42.4 & 0.4 & 36.1 & 0.2 & 59.7 & 0.3 \\
\hline & Personal engagement model & 57.5 & 0.4 & 63.9 & 0.2 & 40.0 & 0.3 \\
\hline
\end{tabular}

Abbreviation: SE, standard error. 
encouraged to 1) ask about safety, 2) report concerns, 3) clean their hands, or 4) tell staff to clean their hands. Simulations predicted that $57.5 \%$ of participants would prefer personal engagement, while $42.4 \%$ would prefer visual engagement (Table 5). The actively engaged segment was more likely to prefer personal engagement (63.9\%), while passively engaged participants would be more likely to choose visual engagement (59.7\%).

\section{Discussion}

This study modeled the safety partnership preferences of health service users. Although DCEs represent a standard approach to the assessment of health service preferences, ${ }^{27}$ we believe this is their first application to the study of safety partnerships. Our findings contribute to research on this topic by estimating the relative value of the different features of multicomponent approaches to safety, demonstrating important individual differences in preferences, and simulating the response of participants to different engagement strategies.

\section{RQ I: What features of safety partnerships are most important to each segment?}

As predicted, participants preferred point of care initiatives such as medication and identity double checks that might contribute to an immediate reduction in personal risk. The administration of medications, for example, represents a significant source of error. ${ }^{52}$ Double-checking medications exerted a stronger influence on choices than any other attribute. This is consistent with studies suggesting that patients rate double checks, and related approaches to the prevention of medication errors, very positively..$^{19,21}$

Hand hygiene campaigns, in contrast, exerted a limited influence on safety partnership choices. Consistent with HYP 2, participants were more likely to choose partnerships prompting patients to wash their hands than those encouraging patients to remind staff to wash their hands. This finding is consistent with research suggesting that patients consider asking staff whether they have cleaned their hands to be less effective than other strategies and themselves to be less likely to participate in these programs than in other risk reduction initiatives. ${ }^{21,53}$ Waterman et a ${ }^{19}$ reported that only $5 \%$ of 2,078 post-discharge inpatients asked nurses or doctors whether they had cleaned their hands. This is consistent with evidence that patients are reluctant to engage in partnerships that might be perceived to challenge the competence of service providers. ${ }^{8}$

\section{RQ 2: Are there segments with different safety partnership preferences?}

Consistent with HYP 3, latent class analysis revealed two segments that might be expected to respond to different safety partnerships. Younger outpatients with higher education were more likely to be members of an actively engaged segment that valued scientific evidence that patients could improve safety. As HYP 4 postulated, they anticipated more benefits to safety partnerships and expressed more confidence in their ability to contribute. As the Theory of Planned Behavior predicts, they were more intent on participating. ${ }^{11}$ They showed a stronger interest in multifaceted approaches to safety education, preferred personal reminders, and advocated complete disclosure of errors. In previous studies, patients with higher activation scores engage in more preventive activities, are more likely to follow treatment protocols, evidence better health outcomes, incur fewer costs, and evaluate care experiences more positively. ${ }^{32}$

Passively engaged participants anticipated fewer benefits to safety partnerships, were less confident in their ability to contribute, and less intent on participating. They preferred that staff give a rationale for safety partnerships and decide whether patients were informed of errors. In comparison to the actively engaged segment, patient representation on safety committees was more important to the passively engaged segment. Passively engaged participants, who may be less comfortable expressing their concerns, may value patient representatives who can speak on their behalf. Participants with immigrant backgrounds, less education, and receiving services as inpatients were more likely to be members of the passively engaged segment. These findings are consistent with studies linking limited education and lower levels of health literacy ${ }^{54}$ to a preference for a less active role in health ${ }^{32,36}$ and safety. ${ }^{3,8,19,55}$

The Theory of Planned Behavior predicts that enhancing self-efficacy should increase the intent to participate. ${ }^{11}$ Although utility values showed that actively engaged participants preferred to read how to make care safer, they also responded positively to a more comprehensive multimodal approach to safety education utilizing readings, videos, and checklists. Passively engaged participants, in contrast, preferred to simply read about safety strategies. However, when the medication and identity double-checks participants valued were included in a multicomponent (readings, videos, and checklists) educational strategy, simulations predicted that $91 \%$ of the passively engaged segment would choose an approach including this active learning option. The results of simulations reveal attitudinal processes via which highly valued attributes 
(medication and identity double checks) compensate for the inclusion of features such as active learning that, while less preferred, may enhance the success of safety partnerships.

\section{RQ 3:To what extent do patients prefer a collaborative approach to safety?}

Consistent with HYP 5, simulations predicted that, rather than delegating responsibility for safety to hospital staff, $97.6 \%$ of our study participants would prefer partnerships between staff and patients. The strength of this finding is consistent with a study reporting that $91 \%$ of a sample of discharged inpatients agreed that patients could contribute to the prevention of health service errors. ${ }^{19}$

\section{RQ 4:What type of engagement strategy do patients prefer?}

HYP 6 postulated that patients would prefer a personal engagement strategy rather than visual safety prompts. Simulations predicted that $57.5 \%$ of the study participants would prefer a personal strategy in which staff engaged patients in safety partnerships. This is consistent with evidence that encouragement by doctors and nurses may increase participation. ${ }^{12}$ In contrast, $42.4 \%$ would prefer an approach with signage reminding patients to ask about safety, report safety concerns, and wash their hands. Preference for a visual communication strategy was strongest in the passively engaged segment. We were surprised by the number of participants predicted to choose a visual strategy. There may be several explanations for this finding. Patients, for example, may perceive signs to be less of a burden to staff than a personal discussion. ${ }^{3}$ In a sample of 277 health care workers, 26\% felt there was insufficient time to encourage patients to ask about hand washing. ${ }^{22}$ Signs may also put less pressure on those who find safety partnerships inconsistent with their perception of the patient's role. In addition to their alignment with the preferences of a significant number of participants, the signage and visual prompts included in a range of health, safety, and infection control initiatives ${ }^{56,57}$ can simultaneously inform patients, family members, staff, and the administrators who are critical to the success of large-scale safety initiatives. ${ }^{39,58}$ Signs can be prepared in multiple languages, use an array of interactive strategies, allow patients to review safety recommendations, and facilitate the positioning of reminders at key points along the health service pathway. ${ }^{59}$ Despite these advantages, effective signage requires careful attention to visual appeal, content, health literacy, and culture; ${ }^{39,60,61}$ evidence regarding its unique contribution to hospital safety is lacking. ${ }^{39}$
Although decision-making processes exerted less influence than many other partnership design attributes, both segments preferred an approach to safety based on shared decisions. Neither segment chose to delegate safety decisions to staff, nor to make these decisions independently. Systematic reviews show that patients are more likely to adhere to health services that are consistent with their preferences. ${ }^{62,63}$ Collaborative decisions, therefore, are more likely to promote the engagement that enhances the patient experience and improves outcome. ${ }^{32}$ Informed decisions require an awareness of the risks safety partnerships might reduce. Risk information exerted an important influence on partnership choices. Both segments preferred that all patients were informed about risks to safety. Awareness of risks is associated with greater confidence in one's ability to prevent medical errors. ${ }^{21}$ Confidence in one's ability to prevent medical errors (self-efficacy), in turn, predicts the intent to act preventively. ${ }^{21}$

Both segments preferred that patients report safety concerns directly to staff and that those conveying safety concerns were thanked and told how the hospital will respond. Although both segments preferred that patients decide whether to give their names when reporting safety concerns, variations in the anonymity afforded those reporting safety concerns exerted relatively little influence on choices.

\section{Limitations}

This study was conducted in Ontario, Canada, a province in which local media coverage of issues such as SARS (severe acute respiratory syndrome) and Avian flu might have altered public perceptions of the risks of hospital-acquired infection and the importance of participation in infection control strategies. ${ }^{64,65}$ Results require replication.

We defined the term staff as doctors, nurses, health care providers, and administrative personnel. The finding that patients are more likely to engage in safety partnerships that may challenge nurses rather than doctors ${ }^{66}$ suggests that future studies should examine preferences for partnerships with different health service providers.

Although the waiting areas in which most surveys were completed may have increased the survey's contextual validity, return rates were reduced: $26 \%$ of those prepared to consider participation were called for appointments before completing the survey. Because this sample loss was related to organizational factors beyond patient control, it did not, in all probability, represent a systematic enrollment bias.

Finally, DCEs have limitations. Study design and analysis are complex, the tradeoffs presented in choice tasks are 
challenging for informants, and the validity of models may be compromised if key attributes are excluded. ${ }^{67}$

\section{Conclusion}

Participants preferred point of contact safety partnerships that might afford an immediate reduction in risk. They valued collaborative decisions supported by information regarding risks, errors, and the benefits of safety partnerships. Health service providers need to ask patients about their goals and preferences, ensure the safety partnerships available reflect the views of different segments, teach the skills needed to participate, enhance self efficacy, and ensure a receptive response when patients participate or raise safety concerns. Respecting individual differences in safety partnership preferences should enhance engagement, improve health outcomes, and contribute to a more positive patient experience. ${ }^{32}$

\section{Acknowledgments}

Portions of this research were supported by the Hamilton Health Sciences Foundation and CANARIE. Charles Cunningham's participation was supported by the Jack Laidlaw Chair in Patient-Centered Health Care at McMaster University Faculty of Health Sciences. The authors acknowledge the research and editorial assistance provided by Stephanie Mielko and Bailey Stewart and the help of the students who participated in this project.

\section{Disclosure}

The authors report no conflicts of interest in this work.

\section{References}

1. Van Den Bos J, Rustagi K, Gray T, Halford M, Ziemkiewicz E, Shreve J. The $\$ 17.1$ billion problem: the annual cost of measurable medical errors. Health Aff (Millwood). 2011;30(4):596-603.

2. Aspden P, Corrigan JM, Wolcott J, Erickson SM. Patient Safety: Achieving a New Standard for Care. Washington DC: The National Academies Press; 2003.

3. Doherty C, Stavropoulou C. Patients' willingness and ability to participate actively in the reduction of clinical errors: a systematic literature review. Soc Sci Med. 2012;75(2):257-263.

4. Davis RE, Sevdalis N, Jacklin R, Vincent CA. An examination of opportunities for the active patient in improving patient safety. J Patient Saf. 2012;8(1):36-43.

5. Wyer M, Iedema R, Hor SY. Editorial: patients: passive subjects or active participants in reducing the spread of infection? J Clin Nurs. 2014; 23(17-18):2385-2386.

6. Longtin Y, Sax H, Leape LL, Sheridan SE, Donaldson L, Pittet D. Patient participation: current knowledge and applicability to patient safety. Mayo Clin Proc. 2010;85(1):53-62.

7. Ward JK, Armitage G. Can patients report patient safety incidents in a hospital setting? A systematic review. BMJ Qual Saf. 2012;21(8): 685-699.
8. Davis RE, Jacklin R, Sevdalis N, Vincent CA. Patient involvement in patient safety: what factors influence patient participation and engagement? Health Expect. 2007;10(3):259-267.

9. Buser GL, Fisher BT, Shea JA, Coffin SE. Parent willingness to remind health care workers to perform hand hygiene. Am J Infect Control. 2013; 41(6):492-496.

10. Burroughs TE, Waterman AD, Gallagher TH, et al. Patients' concerns about medical errors during hospitalization. Jt Comm J Qual Patient Saf. 2007;33(1):5-14.

11. Schwappach DL. Engaging patients as vigilant partners in safety a systematic review. Med Care Res Rev. 2010;67(2):119-148.

12. Davis R, Parand A, Pinto A, Buetow S. Systematic review of the effectiveness of strategies to encourage patients to remind healthcare professionals about their hand hygiene. J Hosp Infect. 2015;89(3): 141-162.

13. McDonald KM, Bryce CL, Graber ML. The patient is in: patient involvement strategies for diagnostic error mitigation. BMJ Qual Saf. 2013;22(suppl 2):ii33-ii39.

14. Greenwald JL, Halasyamani L, Greene J, et al. Making inpatient medication reconciliation patient centered, clinically relevant and implementable: a consensus statement on key principles and necessary first steps. J Hosp Med. 2010;5(8):477-485.

15. Weingart SN, Toth M, Eneman J, et al. Lessons from a patient partnership intervention to prevent adverse drug events. Int J Qual Health Care. 2004;16(6):499-507.

16. Hall J, Peat M, Birks Y, et al. Effectiveness of interventions designed to promote patient involvement to enhance safety: a systematic review. Qual Saf Health Care. 2010;19(5):e10.

17. Entwistle VA, McCaughan D, Watt IS, et al. Speaking up about safety concerns: multi-setting qualitative study of patients' views and experiences. Qual Saf Health Care. 2010;19(6):e33.

18. Vaismoradi M, Jordan S, Kangasniemi M. Patient participation in patient safety and nursing input - a systematic review. J Clin Nurs. 2015; 24(5-6):627-639.

19. Waterman AD, Gallagher TH, Garbutt J, Waterman BM, Fraser V, Burroughs TE. Brief report: hospitalized patients' attitudes about and participation in error prevention. J Gen Intern Med. 2006;21(4): 367-370.

20. Rathert C, Huddleston N, Pak Y. Acute care patients discuss the patient role in patient safety. Health Care Manage Rev. 2011;36(2): 134-144.

21. Hibbard JH, Peters E, Slovic P, Tusler M. Can patients be part of the solution? Views on their role in preventing medical errors. Med Care Res Rev. 2005;62(5):601-616.

22. Longtin Y, Farquet N, Gayet-Ageron A, Sax H, Pittet D. Caregivers' perceptions of patients as reminders to improve hand hygiene. Arch Intern Med. 2012;172(19):1516-1517.

23. Hart $\mathrm{R}$. The effects of a poster in informing and empowering patients in infection prevention and control. J Infect Prev. 2012;13(5):146-153.

24. Longtin Y, Sax H, Allegranzi B, Hugonnet S, Pittet D. Patients' beliefs and perceptions of their participation to increase healthcare worker compliance with hand hygiene. Infect Control Hosp Epidemiol. 2009;30(9): 830-839.

25. Berger Z, Flickinger TE, Pfoh E, Martinez KA, Dy SM. Promoting engagement by patients and families to reduce adverse events in acute care settings: a systematic review. BMJ Qual Saf. 2014;23(7): 548-555.

26. Kemper C, Blackburn C, Doyle JA, Hyman D. Engaging patients and families in system-level improvement: a safety imperative. Nurs Adm $Q$. 2013;37(3):203-215.

27. de Bekker-Grob EW, Ryan M, Gerard K. Discrete choice experiments in health economics: a review of the literature. Health Econ. 2012;21(2): $145-172$.

28. Cunningham CE, Bruce B, Snowdon AW, et al. Modeling improvements in booster seat use: a discrete choice conjoint experiment. Accid Anal Prev. 2011;43(6):1999-2009. 
29. Evans JS. Dual-processing accounts of reasoning, judgment, and social cognition. Annu Rev Psychol. 2008;59:255-278.

30. Caruso EM, Rahnev DA, Banaji MR. Using conjoint analysis to detect discrimination: revealing covert preferences from overt choices. Soc Cogn. 2009;27(1):128-137.

31. Orme BK. Getting Started with Conjoint Analysis: Strategies for Product Design and Pricing Research. 2nd ed. Madison, WI: Research Publishers; 2009.

32. Hibbard JH, Greene J. What the evidence shows about patient activation: better health outcomes and care experiences; fewer data on costs. Health Aff (Millwood). 2013;32(2):207-214.

33. Davis RE, Sevdalis N, Vincent CA. Patient involvement in patient safety: how willing are patients to participate? BMJ Qual Saf. 2011; 20(1):108-114.

34. Davis RE, Sevdalis N, Pinto A, Darzi A, Vincent CA. Patients' attitudes towards patient involvement in safety interventions: results of two exploratory studies. Health Expect. 2013;16(4):e164-e176.

35. Lanza ST, Rhoades BL. Latent class analysis: an alternative perspective on subgroup analysis in prevention and treatment. Prev Sci. 2013; 14(2):157-168.

36. Cunningham CE, Deal K, Rimas H, et al. Using conjoint analysis to model the preferences of different patient segments for attributes of patient-centred care. Patient. 2008;1(4):317-330.

37. Gillespie T. Patient empowerment to improve handwashing compliance by healthcare workers. $J$ Hosp Infect. 2001;49(4):298-299.

38. Hill D. Hand hygiene: are we trying to make the patient the fail-safe system? J Hosp Infect. 2011;79(4):380-381.

39. Gould DJ, Hewitt-Taylor J, Drey NS, Gammon J, Chudleigh J, Weinberg JR. The Clean Your Hands Campaign: critiquing policy and evidence base. J Hosp Infect. 2007;65(2):95-101.

40. McGuckin M, Storr J, Longtin Y, Allegranzi B, Pittet D. Patient empowerment and multimodal hand hygiene promotion: a win-win strategy. Am J Med Qual. 2011;26(1):10-17.

41. Bridges JFP, Hauber AB, Marshall D, et al. Conjoint analysis applications in health - a checklist: a report of the ISPOR good research practices for conjoint analysis task force. Value Health. 2011;14(4): 403-413.

42. Verlegh PWJ, Schifferstein HNJ, Wittink DR. Range and number-oflevels effects in derived and stated measures of attribute importance. Mark Lett. 2002;13(1):41-52.

43. Patterson M, Chrzan K, editors. Partial profile discrete choice: what's the optimal number of attributes? In: Proceedings of the 10th Sawtooth Software Conference; 2003 Apr 15-17; San Antonio, TX. Sequim, WA Sawtooth Software.

44. Johnson RF, Lancsar E, Marshall D, et al. Constructing experimental designs for discrete-choice experiments: report of the ISPOR Conjoint Analysis Experimental Design Good Research Practices Task Force. Value Health. 2013;16(1):3-13.

45. Schwappach DL, Frank O, Davis RE. A vignette study to examine health care professionals' attitudes towards patient involvement in error prevention. J Eval Clin Pract. 2013;19(5):840-848.

46. Cunningham CE, Chen Y, Deal K, et al. The interim service preferences of parents waiting for children's mental health treatment: a discrete choice conjoint experiment. J Abnorm Child Psychol. 2013;41(6): 865-877.

47. Vermunt JK, Magidson J. Latent GOLD® Choice 4.0 User's Manual. Belmont, MA: Statistical Innovations Inc; 2005.
48. Huber J, Orme BK, Miller R. Dealing with product similarity in conjoint simulations. In: Gustafsson A, Herrmann A, Huber F, editors. Conjoint Measurement: Methods and Applications. 4th ed. New York, NY: Springer; 2007:347-362.

49. Orme B. The Sawtooth Software Market Simulator (A supplement to the CBC V. 2.6 Manual). Sequim, WA: Sawtooth Software, Inc; 2006.

50. Orme BK, Huber J. Improving the value of conjoint simulations. Market Res. 2000;12(4):12-20.

51. Nylund K, Asparouhov T, Muthén BO. Deciding on the number of classes in latent class analysis and growth mixture modeling: a Monte Carlo simulation study. Struct Equ Model. 2007;14(4):535-569.

52. Keers RN, Williams SD, Cooke J, Ashcroft DM. Causes of medication administration errors in hospitals: a systematic review of quantitative and qualitative evidence. Drug Saf. 2013;36(11):1045-1067.

53. Schwappach DL, Wernli M. Barriers and facilitators to chemotherapy patients' engagement in medical error prevention. Ann Oncol. 2011; 22(2):424-430.

54. Berkman ND, Sheridan SL, Donahue KE, Halpern DJ, Crotty K. Low health literacy and health outcomes: an updated systematic review. Ann Intern Med. 2011;155(2):97-107.

55. Davis RE, Koutantji M, Vincent CA. How willing are patients to question healthcare staff on issues related to the quality and safety of their healthcare? An exploratory study. Qual Saf Health Care. 2008;17(2): 90-96.

56. Zaghi J, Zhou J, Graham DA, Potter-Bynoe G, Sandora TJ. Improving stethoscope disinfection at a children's hospital. Infect Control Hosp Epidemiol. 2013;34(11):1189-1193.

57. Grundgeiger T, Sanderson PM, Orihuela CB, et al. Prospective memory in the ICU: the effect of visual cues on task execution in a representative simulation. Ergonomics. 2013;56(4):579-589.

58. Duerden BI. Responsibility for managing healthcare-associated infections: where does the buck stop? J Hosp Infect. 2009;73(4):414-417.

59. Ndawula E, Cutter M. "Road signs" approach to hand hygiene. J Hosp Infect. 2001;48(3):242-243.

60. Jenner EA, Jones F, Fletcher BC, Miller L, Scott GM. Hand hygiene posters: motivators or mixed messages? J Hosp Infect. 2005;60(3): 218-225.

61. Jenner EA, Jones F, Fletcher BC, Miller L, Scott GM. Hand hygiene posters: selling the message. J Hosp Infect. 2005;59(2):77-82.

62. Swift JK, Callahan JL, Vollmer BM. Preferences. J Clin Psychol. 2011; 67(2):155-165.

63. Adamson SJ, Bland JM, Hay EM, et al; Preference Collaborative Review Group. Patients' preferences within randomised trials: systematic review and patient level meta-analysis. BMJ. 2008;337:a1864.

64. Young ME, Norman GR, Humphreys KR. Medicine in the popular press: the influence of the media on perceptions of disease. PLoS One. 2008;3(10):e3552.

65. Blendon RJ, Benson JM, DesRoches CM, Raleigh E, Taylor-Clark K. The public's response to severe acute respiratory syndrome in Toronto and the United States. Clin Infect Dis. 2004;38(7):925-931.

66. McGuckin M, Taylor A, Martin V, Porten L, Salcido R. Evaluation of a patient education model for increasing hand hygiene compliance in an inpatient rehabilitation unit. Am J Infect Control. 2004;32(4): $235-238$.

67. Lancsar E, Louviere J. Conducting discrete choice experiments to inform healthcare decision making: a user's guide. Pharmacoeconomics. 2008;26(8):661-677. 


\section{Supplementary material}

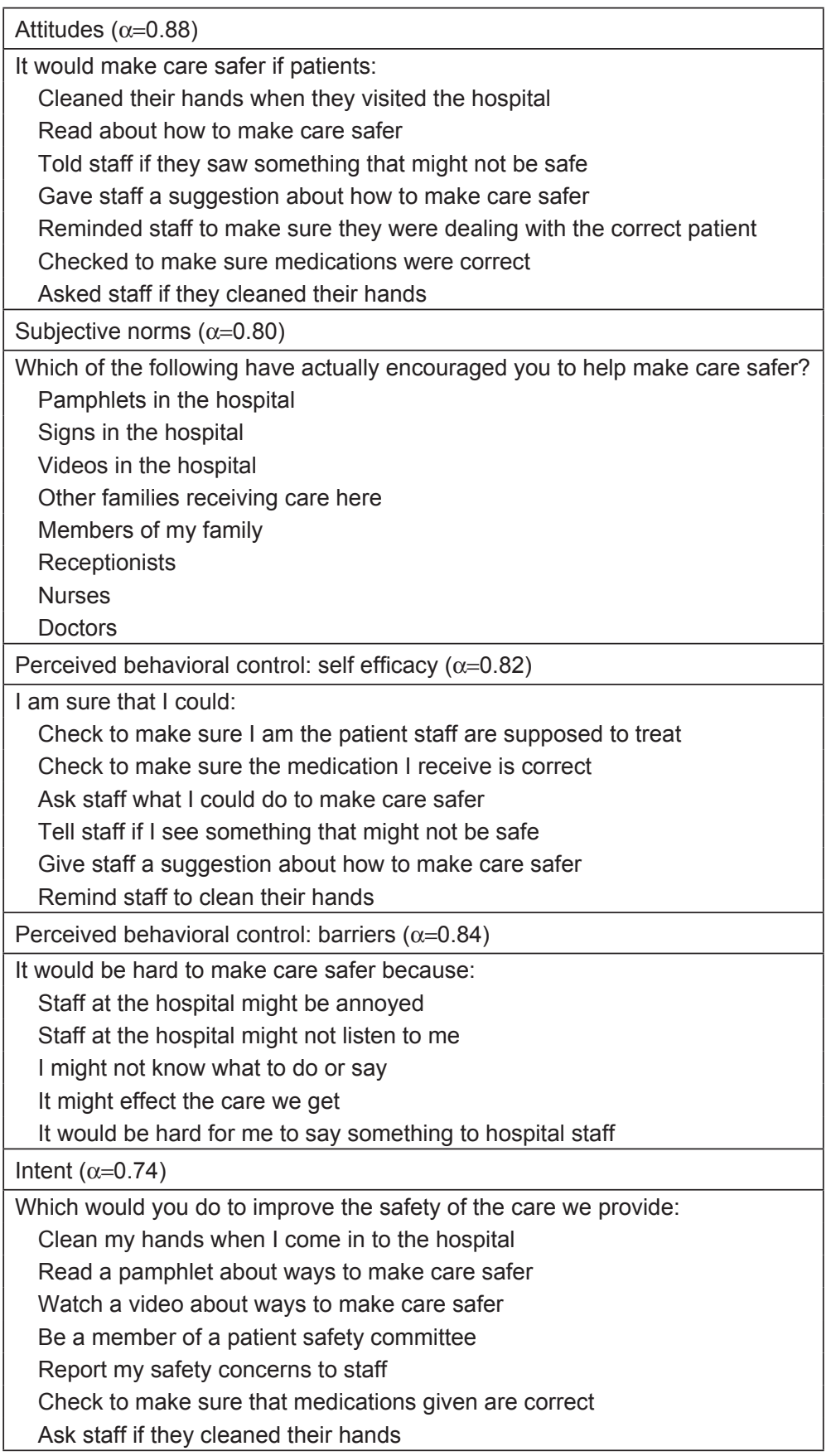

Figure SI Questions from the Safety Partnership Attitudes Questionnaire.

Note: Order of questions within subscales was randomized.

Patient Preference and Adherence

\section{Dovepress}

\section{Publish your work in this journal}

Patient Preference and Adherence is an international, peer-reviewed, open access journal that focuses on the growing importance of patient preference and adherence throughout the therapeutic continuum. Patient satisfaction, acceptability, quality of life, compliance, persistence and their role in developing new therapeutic modalities and compounds to optimize

clinical outcomes for existing disease states are major areas of interest for the journal. This journal has been accepted for indexing on PubMed Central. The manuscript management system is completely online and includes a very quick and fair peer-review system, which is all easy to use. Visit http://www dovepress.com/testimonials.php to read real quotes from published authors. 